\title{
Le dépistage du cancer du poumon par le scanner hélicoïdal est-il justifté?
}

Antoine Flahault, Dominique Costagliola

Le consortium international I-ELCAP (International Early Lung Cancer Action Program) coordonné par Claudia Henschke, radiologue de l'Université Cornell de New-York publie les résultats de la survie à 10 ans de 484 patients ayant eu un diagnostic de cancer du poumon après un dépistage systématique par scanner hélicoïdal à faible dose $^{1}$ [1]. Le dépistage a été réalisé une première fois chez 31567 personnes asymptomatiques à haut risque de cancer du poumon (principalement des fumeurs, avec une médiane de 30 paquets-années ${ }^{2}$ à l'entrée dans l'étude), puis une seconde fois, 7 à 18 mois plus tard, chez 27456 personnes de la cohorte initiale, volontaires pour s'y prêter à nouveau. Les stades précoces représentaient $85 \%$ des 484 cancers détectés, soit 412 patients ayant eu une résection chirurgicale au stade I. La survie de ces derniers était de $88 \%$ à 10 ans, avec un intervalle de confiance à $95 \%$ (74-85), et une mortalité per-opératoire de 0,5\%. Cette survie semble encore meilleure que celle rapportée par le registre nord-américain du cancer du poumon pour les stades I ( $75 \%$ à 8 ans). Alors qu'aujourd'hui, aux États-Unis, $95 \%$ des 173000 personnes dont le diagnostic de cancer du poumon est posé chaque année décèdent de leur cancer, les auteurs suggèrent que le dépistage par le scanner hélicoïdal pourrait prévenir $80 \%$ des décès par cancer du poumon.

${ }^{1}$ Les scanners classiques à rotation séquentielle ont été longtemps utilisés pour l'exploration du corps humain. Avec les progrès technologiques et les recherches effectuées dans le domaine de l'informatique, de l'imagerie et de l'électronique, l'ensemble des constructeurs s'oriente vers une nouvelle génération de machines: le scanner hélicoïdal, encore appelé le scanner à rotation continue. Le scanner hélicoïdal est caractérisé par la rotation continue du couple tube-détecteurs autour d'un lit d'examen, se déplaçant à vitesse constante durant l'acquisition. Le tube à rayons $X$ réalise ainsi un déplacement en hélice, décrivant un cylindre, si l'on se place dans un repère lié à la table.

${ }^{2}$ Unité de mesure conventionnelle mesurant la consommation cumulée de tabac par un fumeur: un paquet-année correspond à la consommation d'un paquet de cigarettes par jour pendant une année. Un patient de 50 ans fumant depuis l'âge de 20 ans un paquet par jour a ainsi fumé 30 paquets-années. un paquet de cigarettes comptant $20 \mathrm{~g}$ de tabac, un paquet-année équivaut à environ $7 \mathrm{~kg}$ de tabac fumé (source : Fédération nationale des centres de lutte contre le cancer).

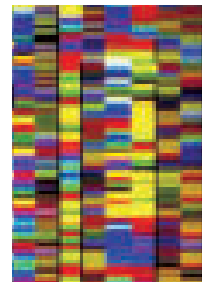

Ces résultats permettent-ils de justifier le dépistage systématique des personnes à haut risque de cancer du poumon? Les auteurs répondent affirmativement, sur deux arguments. D'une part, le taux de cancers du poumon détectés par le scanner hélicoïdal dans leur cohorte est du même ordre que celui de cancers du sein par la mammographie. D'autre part, l'approche médico-économique indique que le rapport coût sur efficacité est comparable dans les deux dépistages.

L'étalon-or de l'évaluation des dépistages des cancers reste cependant l'essai randomisé. Rappelons qu'après huit essais randomisés évaluant l'intérêt de la mammographie dans le dépistage du cancer du sein, des controverses subsistent encore et que certains scientifiques pensent que la qualité de ces essais ne justifie toujours pas la pratique du dépistage systématique [2]. L'étude de C. Henschke et al. n'est pas expérimentale, il s'agit d'une étude observationnelle. L'apport de cette vaste cohorte est considérable, notamment dans la force de propositions et de suggestions qu'elle représente chez les personnes à très haut risque de cancer du poumon. II n'est cependant pas concevable de proposer aujourd'hui de recommander un tel dépistage sans attendre les résultats de plusieurs essais randomisés, de très grande taille, comme ceux 
qui sont en cours de réalisation aux États-Unis (NLST, National Lung Screening Trial) ou en Europe du Nord (Nelson, Nederlands Leuvens Longkanker Screenings Onderzoek). En effet, il est impératif d'éliminer les biais inhérents aux études observationnelles portant sur le dépistage, comme le surdiagnostic et le biais d'avancée de la date du diagnostic. Le surdiagnostic, notamment lié à l'existence de risques compétitifs de décès par d'autres causes, vient du dépistage de tumeurs qui n'auraient jamais connu de manifestations cliniques. De plus certaines des tumeurs pourraient n'avoir jamais progressé et donc jamais été diagnostiquées en l'absence de dépistage. Dans le cas du cancer du sein, des chercheurs suédois ont montré récemment dans une estimation directe, 15 ans après la fin de l'essai de dépistage du cancer du sein organisé à Malmö, que le surdiagnostic était de $10 \%$ chez les femmes de 55 à 69 ans tirées au sort pour faire partie du groupe screening [3]. Les survies prolongées rapportées dans I-ELCAP ne sont-elles pas - pour partie - observées chez des personnes qui n'auraient jamais connu de manifestations bruyantes de leur tumeur de faible grade, voire qui auraient une tumeur indolente qui n'aurait jamais progressé spontanément?

II faut aussi pouvoir mesurer sans biais, dans le cadre d'essais randomisés, les bénéfices, mais aussi les risques, associés à un tel dépistage. Là aussi, dans le cas du dépistage du cancer du sein, on a pu montrer que le coût associé aux faux positifs du screening pouvait être extrêmement élevé [4], sans parler de l'anxiété engendrée chez les femmes lorsqu'existe un délai de plusieurs mois entre le dépistage et le diagnostic final d'absence de cancer. La mortalité opératoire est de $0,5 \%$ dans l'étude I-ELCAP. Lorsque I'on sait qu'un essai pilote de dépistage du cancer du poumon par le scanner hélicoïdal à faible dose, Dépiscan, mené en France, a conduit à détecter un nodule pulmonaire initialement suspect de malignité chez près de la moitié des sujets volontaires asymptomatiques à risque, on peut redouter que les risques liés aux investigations consécutives au dépistage ne viennent en obérer les bénéfices. L'espoir suscité par la dernière publication de I-ELCAP renforce l'importance et l'urgence de voir se conduire plusieurs essais randomisés de grande taille et de grande qualité méthodologique sur le sujet. Ces essais doivent inclure l'évaluation à long terme du surdiagnostic et l'évaluation des coûts matériels et humains des faux positifs, en plus de l'évaluation du bénéfice en termes de survie. $\diamond$

Is the tracking of the lung cancer

by the helicoid scanner justified?

\section{RÉFÉRENCES}

1. The International Early Lung Cancer Action Program Investigators. Survival of patients with stage I lung cancer detected on CT Screening. $N$ Engl J Med 2006 ; 355 : 1763-71.

2. Charatan F. US panel finds insufficient evidence to support mammography. Br Med J $2002 ; 324: 255$.

3. Zackrisson S, Andersson I, Janzon L, Manjer J, Garne JP. Rate of overdiagnosis of breast cancer 15 years after end of Malmö mammographic screening trial: follow-up study. Br Med J 2006 ; 332: 689-92.

4. Lidbrink $\varepsilon$, $\varepsilon l$ fving J, Frisell J, Jonsson $\varepsilon$. Neglected aspects of false positive findings of mammography in breast cancer screening: analysis of false positive cases from the Stockholm trial. Br Med J 1996 ; $312: 273-6$.

\section{TIRÉS À PART}

D. Costagliola

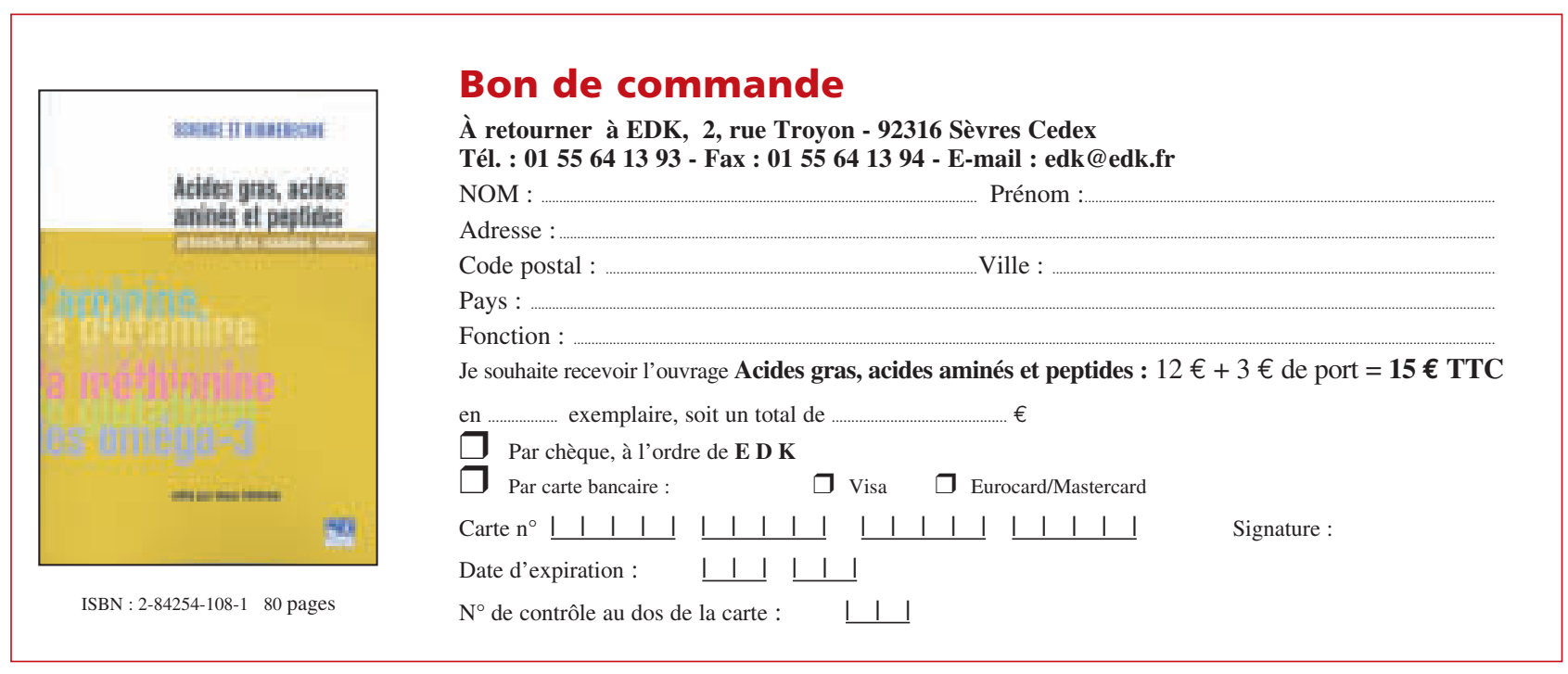

